Int. J. Dev. Biol. 54: 609-615 (2010)

doi: $10.1387 / \mathrm{ijdb} .092929 \mathrm{ks}$

\title{
XRASGRP2 is essential for blood vessel formation during Xenopus development
}

\author{
KAN SUZUKI ${ }^{1}$, SHUJI TAKAHASHI ${ }^{2}$, YOSHIKAZU HARAMOTO ${ }^{2}$, YASUKO ONUMA ${ }^{3}$, KENTARO NAGAMINE ${ }^{4}$, \\ KOJI OKABAYASHI ${ }^{5,7}$, KOHEI HASHIZUME ${ }^{6}$, TADASHI IWANAKA ${ }^{1}$ and MAKOTO ASASHIMA ${ }^{*, 3,5,7}$ \\ ${ }^{1}$ Department of Pediatric Surgery and Oncology, Graduate School of Medicine, and ${ }^{2}$ Center for Structuring \\ Life Science, Graduate School of Arts and Sciences, The University of Tokyo, ${ }^{3}$ Organ Development Re- \\ search Laboratory, National Institute of Advanced, Industrial Sciences and Technology (AIST), ${ }^{4}$ Laboratory \\ of Biochemistry, Hiroshima International University, ${ }^{5}$ ICORP Project (JST), Graduate School of Arts and \\ Science, The University of Tokyo, ${ }^{6}$ Tokyo-West Tokushukai Hospital and ${ }^{7}$ Department of Life Sciences \\ (Biology), Graduate School of Arts and Science, The University of Tokyo, Japan
}

\begin{abstract}
Ras guanyl nucleotide-releasing protein 2 (RASGRP2), one of the Ras guanine exchange factors, is implicated as a critical regulator of inside-out integrin activation in human lymphocytes, neutrophils and platelets. However, the activities of this protein in endothelial cells remain unclear. In the current study, we identify a physiological function in blood vessel formation for XRASGRP2, which is the Xenopus ortholog of mammalian RASGRP2. XRASGRP2 overexpression induced ectopic vascular formation, and XRASGRP2-knockdown embryos showed delayed vascular development. We also investigated the upstream signaling of XRASGRP2 in endothelium formation. XRASGRP2 expression was up-regulated in the presence of VEGF-A and down-regulated following VEGF-A depletion. XRASGRP2 knockdown abolished the ectopic induction of endothelial cells by VEGF-A in the posterior ventral blood island. These results suggest that XRASGRP2 is essential for vascular formation during Xenopus development.
\end{abstract}

KEY WORDS: XRASGRP2, Xenopus laevis, VEGF-A, RASGRP, vasculogenesis

\section{Introduction}

Vascular and hematopoietic cells are thought to arise from a common progenitor, the hemangioblast. In Xenopus, primitive red blood cells are produced exclusively in the ventral blood island (VBI), which is functionally equivalent to the extra-embryonic yolk sac blood island in mammals. The embryonic endothelial cells arise synchronously with the primitive red blood cells in the VBI. The close spatial and temporal relationships between the blood cells and endothelial cells support the hypothesis that they have a bipotential precursor, the hemangioblast (Sabin, 1920; Murray, 1932). However, the developmental mechanism underlying the differentiation of endothelial and primitive blood cells from the hemangioblast remains unclear.

Vascular endothelial growth factors (VEGFs) are key regulators in vasculogenesis and angiogenesis (Ferrara etal., 2003). In particular, VEGF-A is involved in the regulation of processes required for angiogenesis, i.e., endothelial cell activation, proliferation, migration, and tubule formation (Ferrara et al., 2003). In Xenopus, the VEGF$A$ gene is alternatively spliced to produce the VEGF122, VEGF170, and VEGF190 isoforms, which are equivalent to murine VEGF120,
VEGF164, and VEGF188, respectively (Cleaver et al., 1997). Ectopic expression of VEGF122changes the architecture of the developing vascular network (Cleaver et al., 1997). Over-expression of VEGF170 induces the inhibition of expression of the hematopoietic genes $\alpha$-globinand GATA-1 in the posterior blood island, as well as the excessive production of endothelial cells (Koibuchi et al., 2006).

Signaling through Ras is one of the intracellular pathways downstream of VEGF stimulation (Doanes et al., 1999; Hood et al., 2003; Meadows et al., 2001). Genetic ablation of either SOS, which encodes a Ras guanine nucleotide exchange factor, or NF1, which encodes a Ras GTPase-activating protein, results in cardiovascular defects (Brannan et al., 1994; Henkemeyer et al., 1995; Wang et al., 1997). K-ras-deficient mice die having multiple defects, including defects of the hematopoietic and cardiovascular systems (Johnson et al., 1997; Koera et al., 1997). Targeted deletion of NF1 in

\footnotetext{
Abbreviations used in this paper: RASGRP, Ras guanyl nucleotide-releasing protein; VBI, ventral blood island; VEGF, vascular endothelial growth factor; Xmsr, Xenopus mesenchyme associated serpentine receptor.
}

*Address correspondence to: Dr. Makoto Asashima. 3-8-1 Komaba, Meguro-ku, Tokyo 153-8902, Japan. Fax: +81-3-5454-4330.

e-mail: asashi@bio.c.u-tokyo.ac.jp

Accepted: 7 May 2009. Final author-corrected PDF published online: 3 June 2009. Edited by: Makoto Asashima.

ISSN: Online 1696-3547, Print 0214-6282 
endothelial cells leads to multiple cardiovascular defects (Gitler et al., 2003). The small GTPase Rap1b is required for normal angiogenesis and plays a role in the regulation of pro-angiogenic signaling in endothelial cells (Chrzanowska-Wodnicka et al., 2007).

RasGRP2/CalDAG-GEFI is a member of the CalDAG-GEF/ RasGRP family of intracellular signaling molecules involved in the activation of the Ras superfamily (Kawasaki et al., 1998; Springett etal., 2004). RasGRP2 contains binding sites for $\mathrm{Ca}^{2+}$ and DAG, and a GEF domain that predominantly activates Rap1 (Kawasaki et al., 1998). Recently, RasGRP2 was identified as a critical regulator of inside-out integrin activation in human T lymphocytes, neutrophils, and platelets (Pasvolsky et al., 2007). RasGRP3 is expressed in embryonic blood vessels and newly formed vessels during pregnancy and tumorigenesis in adults. RasGRP3 expression is upregulated by VEGF stimulation of endothelial cells (Roberts et al.,

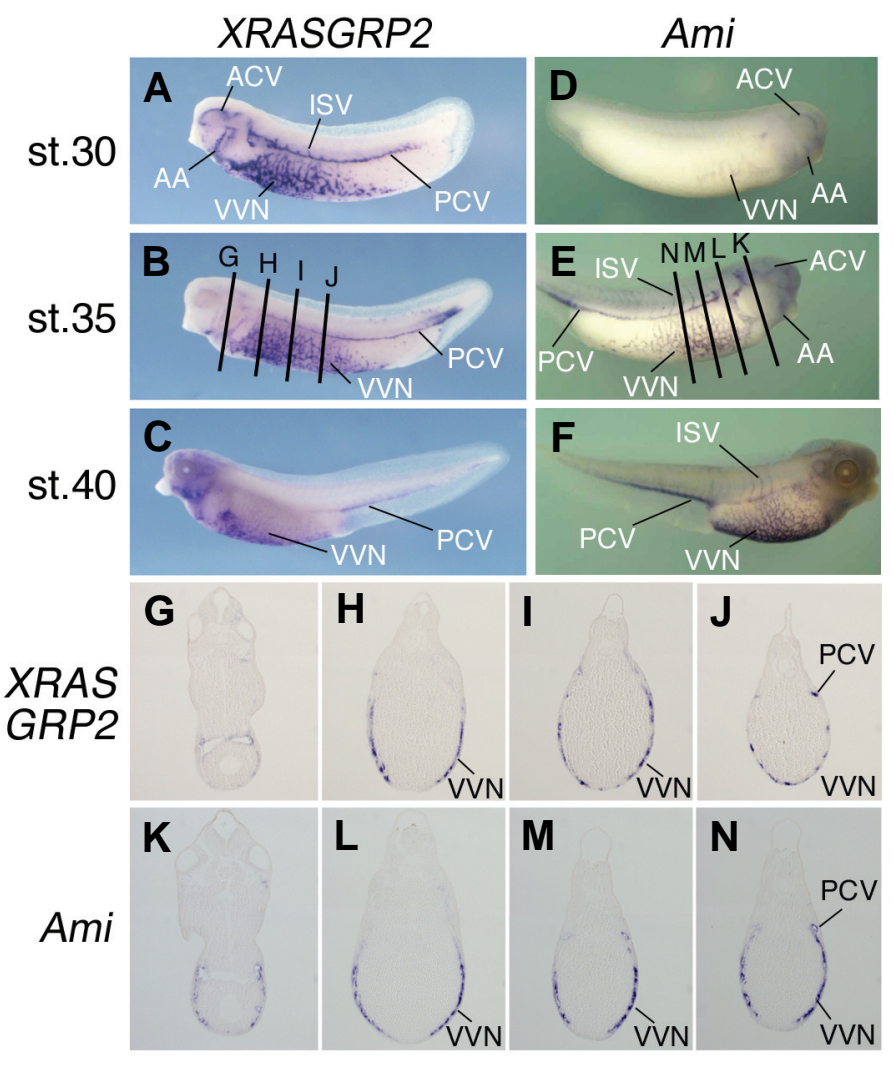

2004). In Xenopus, XRASGRP2is expressed in the vascular region of the embryo (Nagamine et al., 2008).

In the present study, we reveal the role of XRASGRP2 in Xenopus vascular development by showing that: 1) over-expression of $X R A S G R P 2$ induces ectopic endothelial cell differentiation; 2) $X R A S G R P 2-k n o c k d o w n$ embryos show reduction or delay of endothelial cell differentiation; and 3) XRASGRP2 expression is induced by VEGF-A signaling. Our findings indicate that XRASGRP2 is essential for vascular development in downstream of VEGF-A signaling in Xenopus embryos.

\section{Results}

\section{XRASGRP2 expression is restricted in Xenopus developing vessels}

Previously, it was shown that Xenopus laevis RASGRP2 (XRASGRP2) is expressed in the vascular regions of stage 35 embryos (Nagamine etal., 2008). We examined in detail XRASGRP2 expression during vascular development. XRASGRP2mRNA was found to be expressed in vascular regions, such as the anterior cardinal vein (ACV), aortic arch (AA), intersomitic vein (ISV), posterior cardinal vein (PCV), and vascular vitelline network (VVN) at stage 30 (Fig. 1A). The expression levels of XRASGRP2mRNA in the ACV, $A A$, and ISV were reduced at stage 35 (Fig. 1B). At stage 40, $X R A S G R P 2$ expression was restricted to the PCV and VVN (Fig. $1 \mathrm{C})$. In contrast, the expression of $A m i$, which is a vascular-specific gene, was detected in the ACV, AA, and VVN, but not in the ISV and PCV, at stage 30 (Fig. 1D). At stage 35, expression of Ami was detected in the ACV, AA, ISV, PCV, and VVN (Fig. 1E). These results

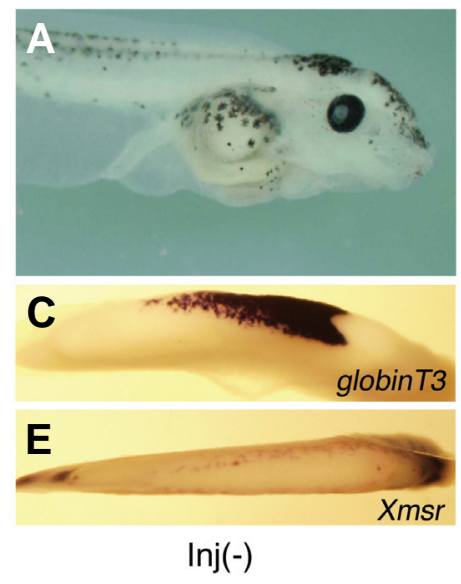

Fig. 1 (Left). XRASGRP expression precedes Ami expression. (A) XRASGRP2 transcripts localized in the anterior cardinal vein (ACV), aortic arch (AA), intersomitic veins (ISV), posterior cardinal veins (PCV), and vascular vitelline network (VVN) at stage 30. (B) XRASGRP2 expression is detected in the PCV and VVN at stage 35. Lines indicate the positions of the sections shown in (G-J). (C) At stage 40, XRASGRP2 expression is restricted to the PCV and VVN. (D) The expression of Ami is weakly detected in the ACV, AA, and VVN at stage 30. (E) Ami expression is evident in the ACV, AA, $I S V, P C V$, and VVN at stage 35. Lines indicate the positions of the sections shown in (K-N). (F) Ami expression is detected continuously in the PCV, ISV, and VVN until stage 40. (G-J) Histologic section of the embryo shown in (B). (K-N) Histologic section of the embryo shown in (E). Both XRASGRP2 and Ami are expressed in the endothelial cells (PCV and VVN).

Fig. 2 (Right). Ectopic expression of XRASGRP2 affects vascular formation and induces edema. (A) An uninjected control embryo at stage 43. (B) An embryo in which $1 \mathrm{ng}$ of XRASGRP2 mRNA was injected into the dorsal vegetal blastomeres (DV) at the 8-cell stage. The embryo shows edema. (C-F) Whole-mount in situ hybridization for a hematopoietic marker, globin T3, and an endothelial marker, Xmsr, at stage 31. (C) The expression of globin T3 in an uninjected control embryo (ventral view). (D) Expression of globin T3 in an embryo that was co-injected with XRASGRP2 and $\beta$ galactosidase ( $\beta$-gal) into the ventral vegetal blastomeres (VV) at the 8-cell stage. The expression of globin T3 is abolished at the injection site in the VBI. (E) The expression of Xmsr in an uninjected control embryo (ventral view). (F) The expression of Xmsr in an embryo that was co-injected with XRASGRP2 and $\beta$-gal into the VV. Ectopic expression of Xmsr is evident at the injection site in the VBI. Arrowheads indicate Xmsr-positive cells. 
A

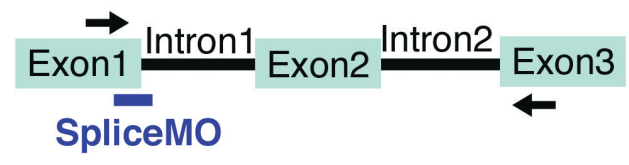

B

(-) un c aS bS S

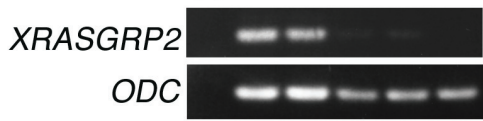

D $\quad \ln (-)$

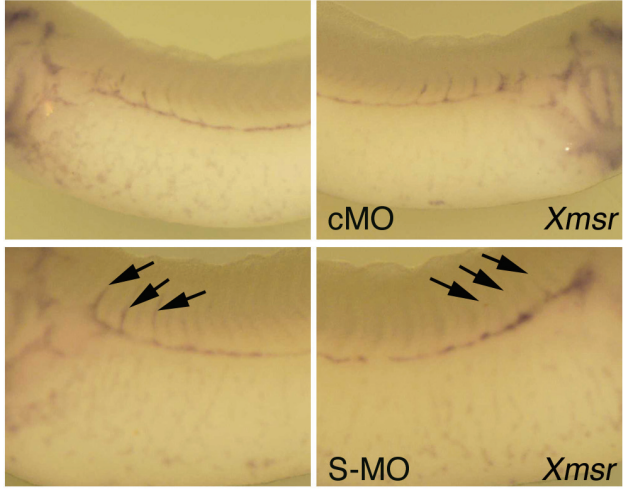

$\mathbf{F}$
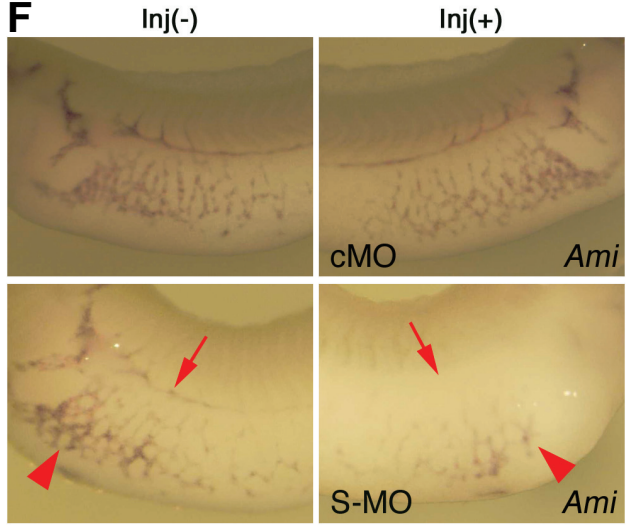

H
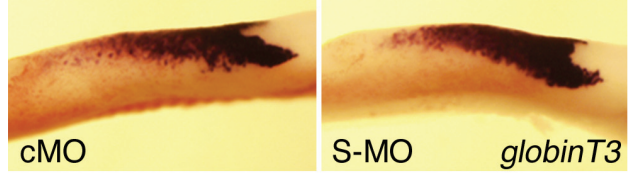

C
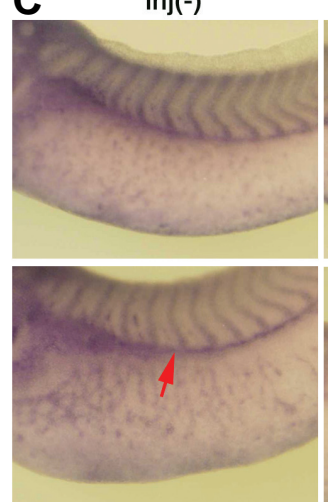

E
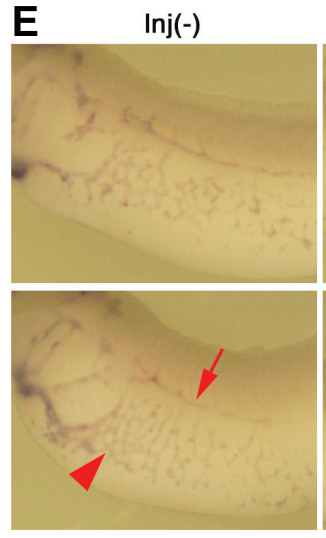

cMO
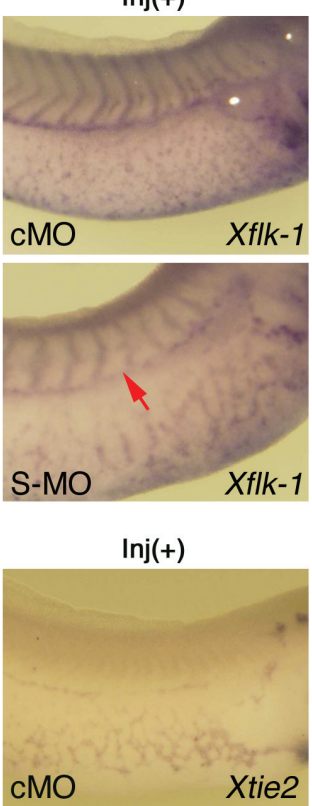

G
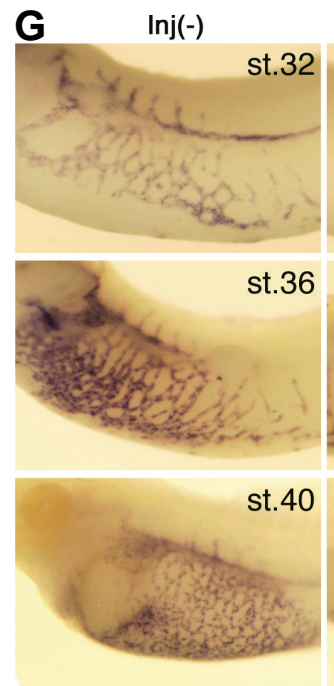
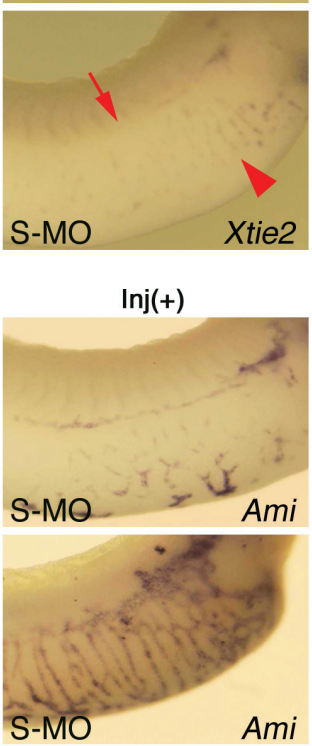

Ami

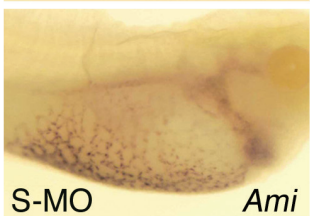

Fig. 3. XRASGRP2 depletion results in aberrant development of blood vessels. (A) Schematic model for the splice inhibition antisense morpholino oligonucleotides (S-MOs). The binding site of $\mathrm{MO}$ is represented by a bolded blue line. Arrows indicate the primers used in the $R T-P C R$ to examine the efficacies of the S-MOs. (B) The control MO (c, 40 ng), aS$M O(a S, 40 \mathrm{ng}), b S-M O(b S, 40 \mathrm{ng})$, and $S$ MO (S, 40 ng, comprising 20 ng aS-MO plus $20 \mathrm{ng}$ bS-MO) were injected into 2cell-stage embryos, and the embryos were analyzed by RT-PCR at stage 30. The presence of the 312-bp band indicates amplification of the normally spliced $m R N A$. The intensity of this band is reduced in both the aS-MO-injected and bSMO-injected embryos, as compared to the uninjected embryos and control MOinjected embryos, and this band is not detected for the S-MO-injected embryos. This indicates that the S-MO-injected embryos do not produce a functional XRASGRP2 protein. '-', Sample without reverse transcriptase; 'un', uninjected embryos. (C-F) Expression patterns of blood vessel marker genes. The 2-cell-stage embryos were injected with the control MO (40 ng) or S-MO (40 ng) into one blastomere (corresponding to the future right-hand side), and harvested at stage 31. The injected sides are indicated as $[I n j(+)]$ and the uninjected sides are indicated as [Inj(-)]. The expression levels of Xflk-1 in the PCV (C, red arrows) and of Xmsr in the ISV (D, black arrows) are diminished in the S-MO-injected side. The expression levels of Xtie2 (E) and Ami (F) in the PCV (red arrows) and VVN (red arrowheads) are diminished in the S-MOinjected side. The expression level of Ami $(F)$ is greatly reduced in the S-MO-injected side. No differences are seen in the control MO-injected embryos. (G) The expression of Ami in VVN is gradually mitigated in the S-MO-injected side. (H) Expression of globin T3 in the control MO-injected embryos. S-MO injection does not affect the level of globin T3 expression. suggest that the expression of $X R A S G R P 2$ is transient and occurs earlier than the expression of $A m i$ in developing vascular regions. Examination of the sections of the stage 35 embryos showed that both XRASGRP2and Amiwere strongly expressed in the VVN and PCV (Fig. 1 G-N). The expression of $A$ miwas reduced in the ACV and AA at stage 40 (Fig. 1F).

\section{Over-expression of XRASGRP2 induces ectopic expression of Xmsr}

We examined the function of XRASGRP2 in vascular development. XRASGRP2 mRNA and $\beta$-galactosidase ( $\beta$-gal) mRNA were injected into dorsal-vegetal (DV) or ventral-vegetal (VV) blastomeres at the 8-cell stage. DV and VV blastomeres contain components of future VBI cells. The injected embryos showed the edema phenotype at the tail-bud stage, and this phenotype was more severe at stage $43(75 \%, n=56)$, as compared to uninjected control embryos $(6 \%, n=72)$ (Fig. $2 A, B)$. It was assumed that over-expression of XRASGRP2influences cardiovascular development. Whole-mount in situ hybridization revealed that the expression of globin T3was suppressed in the VBI (Fig. 2D), and that ectopic expression of $X m s r$ was induced (Fig. $2 F$ ) in the $X R A S G R P 2-i n j e c t e d$ embryos (Fig. 2 C,E). Xmsr, which is the 

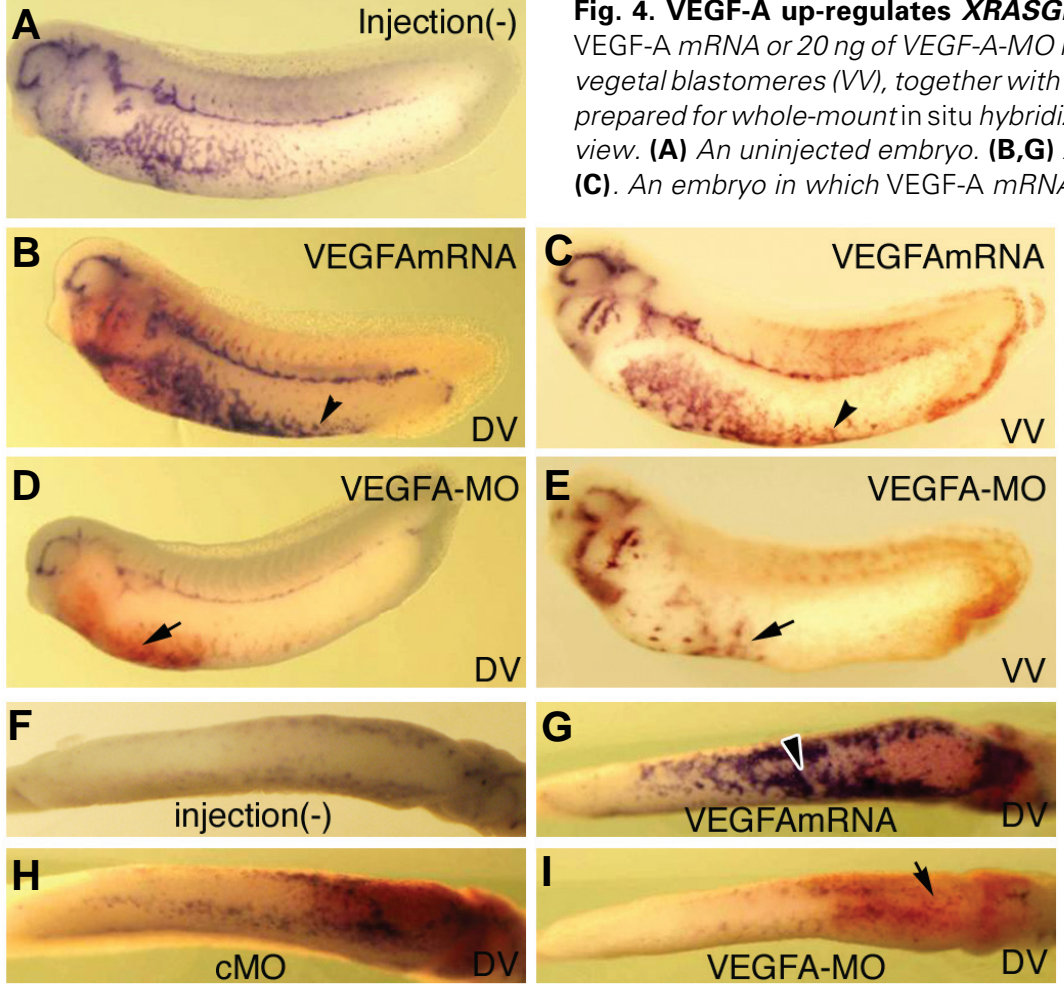

VEGFA-MO

Fig. 4. VEGF-A up-regulates XRASGRP2 expression. Embryos were injected with either 1 ng of VEGF-A mRNA or 20 ng of VEGF-A-MO into the two dorsal-ventral blastomeres (DV) or the two ventralvegetal blastomeres (VV), together with $200 \mathrm{pg}$ of $\beta$-gal $m R N A$, at the 8-cell-stage. These embryos were prepared for whole-mount in situ hybridization of XRASGRP2 at stage 32. (A-E) Lateral view. (F-I) Ventral view. (A) An uninjected embryo. (B,G) An embryo in which VEGF-A mRNA was injected into the DV. (C). An embryo in which VEGF-A mRNA was injected into the VV. (D,I) An embryo in which VEGF-AMO was injected into the DV. (E) An embryo in which VEGFA-MO was injected into the VV. (F) An uninjected embryo. (H) An embryo in which the control MO (20 ng) was injected into the DV. Black arrows indicate inhibition of XRASGRP2 expression in the $V V N(D, E)$ and $V B I$ (I). Black arrowheads indicate ectopic expression of XRASGRP2 in the VVN $(B, C)$ and $\mathrm{VBI}(\mathrm{G})$.

bS-MO co-injected embryos (Fig. 3B, lane S). These results indicate that aS-MO and bS-MO effectively inhibit XRASGRP2 gene splicing and production of the intact protein. To investigate the role of $X R A S G R P 2$ in normal vascular development, a mixture of XRASGRP2-MOs (S-MO) was injected into one side of the 2-cell-stage embryos. The embryos were fixed at stage 31 , to analyze the expression levels of the vascular-specific marker genes $X f / k-1$, $X m s r, X$ tie2, and Ami (Fig. 3 C-F). The expression levels of $X f / k-1$ and $X m s r$ were reduced in the PCV and ISV, respectively (Fig. 3 C,D, arrows), and the expression levels of $X$ tie2 and $A m i$ were suppressed in the PCV and VVN (Fig. $3 \mathrm{E}, \mathrm{F}$, arrows and arrow-

Xenopus homolog of the G-protein-coupled receptor APJ, functions as the apelin receptor. Xmsris expressed by endothelial cells (Devic et al., 1996; Inui et al., 2006). These results indicate that the over-expression of XRASGRP2 alters vascular and hematopoietic cell fates, leading to the replacement of blood cells with endothelial cells.

\section{Knockdown of XRASGRP2 disrupts vascular development}

The antisense XRASGRP2-MOs (aS-MO and bS-MO) were designed to block splicing at the first exon/intron boundaries (Fig. 3A, see Materials and Methods). These MOs inhibit the normal splicing of XRASGRP2 pre-mRNA, resulting in the production of a truncated protein that lacks the functional domain. RT-PCR analysis revealed that the level of the normally spliced transcript (312-bp band) was reduced in aS-MOor bS-MO-injected embryos (Fig. 3B, lanes aS and bS). Normally spliced transcripts were not detected in the aS-MO and heads) of the S-MO-injected sides. The suppression of Ami expression at the S-MO-injected side was gradually mitigated bryo showed no significant changes in globin T3 expression under the conditions used in the present study (Fig. $3 \mathrm{H}$ ).

\section{VEGF-A signaling regulates the expression of XRASGRP2 and acts through XRASGRP2 in vascular development}

VEGF-A is a key factor in vasculogenesis and induces endothelial gene expression. A functional analysis of VEGF in Xenopus embryos was performed by injecting VEGF-A mRNA or VEGF-A-MO into dorsal-vegetal blastomeres or ventralvegetal blastomeres at the 8-cell stage (Koibuchi et al., 2006). We then examined the impact of signaling upstream of XRASGRP2on vascular development. VEGF-AmRNA or VEGFA-MO was injected into dorsal-vegetal blastomeres or ventralvegetal blastomeres, together with $\beta-g a / \mathrm{mRNA}$, at the 8-cell as the embryos developed (Fig. 3G). The S-MO-injected em-

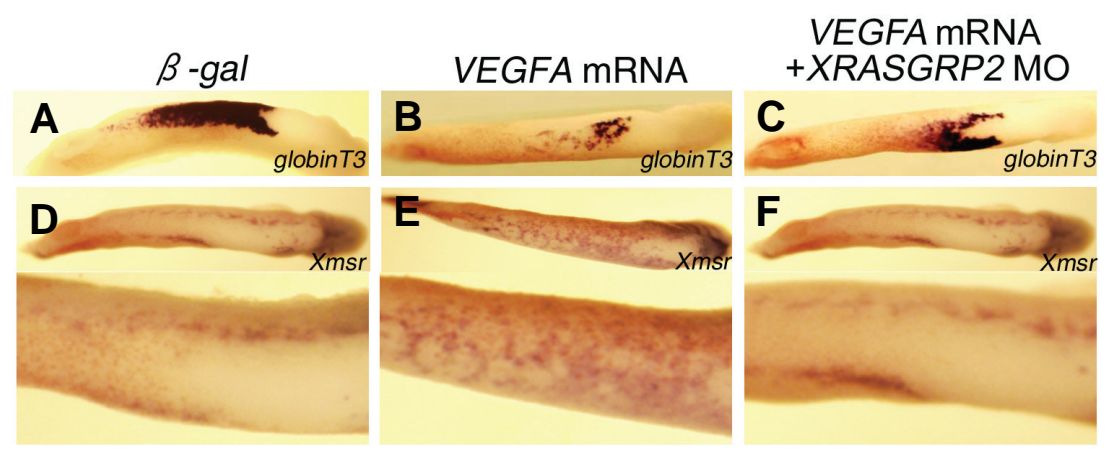

Fig. 5. RasGRP2 mediates VEGF-A signaling. Embryos were injected with $200 \mathrm{pg}$ of $\beta$-gal mRNA $(A, D), 1 \mathrm{ng}$ of VEGF-A mRNA (B,E) or 1 ng of VEGF-A mRNA plus 40 ng of XRASGRP2S-MO $(C, F)$ into two ventral-vegetal blastomeres at the 8-cell stage. The embryos were cultured until stage 31, for whole-mount in situ hybridization analysis. (A-C) Expression patterns of globin T3. (D-F) Expression patterns of Xmsr. VEGF-A inhibits globin T3 expression in the VBI. Higher-magnification images showing the Xmsr expression patterns in the $\mathrm{VB} /$ region are shown (lower panels). (B). VEGF-A-mediated suppression of globin T3 expression is partially rescued by co-injection of the XRASGRP2 S-MO (C). VEGF-A induces ectopic Xmsr expression in the VBI (E). VEGF-A-induced ectopic expression of Xmsr is partially rescued by co-injection of the XRASGRP2 S-MO (F). 


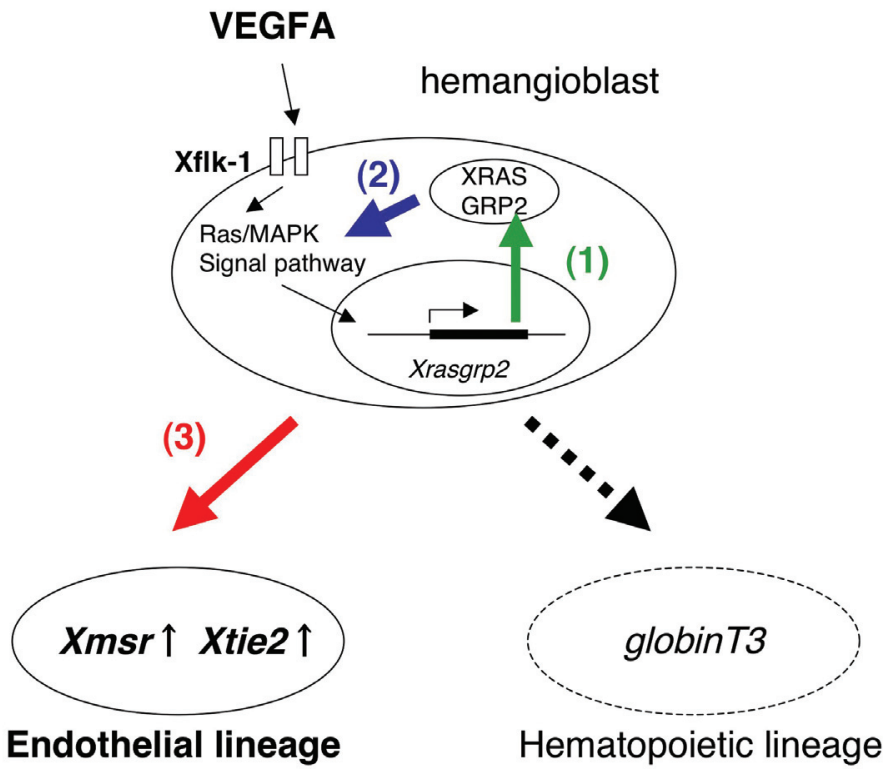

Fig. 6. A model for XRASGRP2 function in hemangioblast cells. (1) The expression of XRASGRP2 is induced by VEGF-A in hemangioblast cells. (2) XRASGRP2 reinforces the VEGF-A/Ras signal pathway. (3) The stimulated hemangioblast cells differentiate to endothelial cells that express Xmsr and Xtie2. In this case, differentiation to the hematopoietic lineage is suppressed.

stage. The number of XRASGRP2-positive cells increased in the area that was injected with $V E G F-A$ mRNA (Fig. $4 \mathrm{~B}, \mathrm{C}, \mathrm{G}$ ). In contrast, XRASGRP2 expression was reduced in the $\mathrm{VVN}$ at stage 32 in the VEGF-A-MO-injected embryos (Fig. 4 D,E,I, arrow). These results indicate that VEGF-A induces $X R A S G R P 2$ expression, and that VEGF-A is required for XRASGRP2 expression. In the $V E G F-A$ mRNA-injected embryos, ectopic induction of endothelial cells that expressed Xmsrand inhibition of globin T3expression were observed (Fig. $5 \mathrm{~B}, \mathrm{E}$ ). When VEGF-A mRNA and the XRASGRP2 S-MO were co-injected, the ectopic expression of $X m s r$ was decreased (Fig. 5 E,F) and the expression of globin T3was partially rescued (Fig. 5 B,C). These results suggest that VEGF signaling acts through XRASGRP2 in vascular development.

\section{Discussion}

In humans, RasGRP2 has been identified as a critical regulator of inside-out integrin activation in T lymphocytes, neutrophils, and platelets (Pasvolsky et al., 2007). In adult rodents, RasGRP2 is expressed in platelets, megakaryocytes, and neutrophils within the hematopoietic system, as well as in neurons, especially in the striatum of the basal ganglia (Crittenden et al., 2004; Kawasaki et al., 1998). The expression of XRASGRP2 in the developing vascular system was reported in Xenopus (Nagamine et al., 2008). This XRASGRP2 expression coincided with that of $X f / k-1$ and $X m s r$, and occurred earlier than the expression of $A m i$. $X R A S G R P 2$ expression was found to be transient in the developing vascular regions (Fig. 1) (Cleaver et al., 1997; Devic et al., 1996; Inui and Asashima, 2006). These results indicate that $X R A S G R P 2$ plays a role in the early phase of vasculogenesis.
In the present study, the edema phenotype was observed for both the up-regulation and down-regulation of XRASGRP2. It was reported that VEGF-A overexpression induced edema (Koibuchi et al., 2006). VEGF-A overexpression led to the formation of ectopic blood vessels and reduced blood circulation. In contrast, $c$-myc-knockdown embryos showed decreased vessel formation and had the edema phenotype (Rodrigues et al., 2008). These results indicate that an appropriate level and precise timing of blood vessel formation are required for normal development.

The XRASGRP2-knockdown embryos showed the edema phenotype, which may be due to circulation problems (data not shown) and inhibition of endothelial gene expression (Fig. $3 \mathrm{C}-\mathrm{F}$ ). This outcome indicates that XRASGRP2 is necessary for endothelial differentiation. However, the delayed vessel formation observed on the XRASGRP2-depleted side was gradually mitigated (Fig. 3G). This indicates that some other molecules partly compensate for the lack of XRASGRP2 function. It has been reported that RasGRP3 is expressed in endothelial cells in the developing mouse embryo, although a loss-of-function mutation in RasGRP3did not affect mouse embryo viability (Roberts et al., 2004). Genetic ablation of Sos in the mouse resulted in death at mid-gestation, with evidence of cardiovascular and yolk sac defects (Qian etal., 2000; Wang etal., 1997). These orthologs are candidates for the factors that compensate for the loss of XRASGRP2 function in Xenopus vasculogenesis.

VEGF plays a central role in vascular development (Ferrara et al., 2003). VEGF-A signaling is necessary and sufficient for promoting early endothelial differentiation in Xenopus. Overexpression of VEGF-Acaused ectopic expression of XRASGRP2 (Fig. 4 B,C,G), similar to that of Xmsrand Xtie2(Fig. 5, Koibuchi et al., 2006). In addition, XRASGRP2 expression was suppressed in VEGF-A-disrupted embryos (Fig. 4). The over-expression of $X R A S G R P 2$ resulted in ectopic expression of $X m s r$, similar to the over-expression of $V E G F-A$ (Figs. 2 and 4). These results indicate that VEGF-A is the endogenous upstream factor of XRASGRP2 in Xenopus endothelial cell differentiation.

The XRASGRP2 S-MO inhibited the ectopic expression of the genes induced by VEGF-A over-expression (Fig. 5), which suggests that XRASGRP2 is necessary for VEGF-A to induce endothelial cell differentiation. Therefore, the expression of XRASGRP2 is regulated by VEGF-A signaling, and the induced XRASGRP2 facilitates or maintains VEGF-A signaling for endothelial cell differentiation. We propose a model for the function of RASGRP2 in vasculogenesis (Fig. 6). In hemangioblasts, the VEGF signal induces XRASGRP2expression. XRASGRP2 directs the cell fate towards the endothelial lineage. Since RasGRP is an activator of members of the small GTPase family, such as Ras and Rap1, these molecules are candidate targets of RASGRP2 in endothelial differentiation. Further studies are required to elucidate the role of XRASGRP2 in VEGF signal transduction and to identify the target molecule of XRASGRP2 in vasculogenesis.

\section{Materials and Methods}

\section{Plasmid constructs}

The following constructs were generated for in vitro RNA synthesis: pCS2P-XRASGRP2, which contains the ORF of Xenopus RASGRP2, and pCS2-VEGF-A, which contains the ORF of Xenopus VEGF-Ab (isoform 4, VEGF168, DQ481238). The plasmids were generated by PCR amplification using the Phusion High Fidelity PCR Kit (Finnzymes, Fin- 
land) followed by subcloning into the pCS2+ vector (Turner and Weintraub, 1994).

\section{Morpholino oligonucleotide design and validation}

We obtained the sequences of the XRASGRP2a and XRASGRP2b genes of $X$. laevis (corresponding to the pseudo-tetraploid genome) from the database. Through prediction using the Xenopus tropicalis genomic sequence, the following common primers for exons 1 and 3 of XRASGRP2a and $X R A S G R P 2 b$ were generated:

forward, 5'-CTGATCTTGATAAGGGTCTCACCA-3';

reverse, 5'-CTGTTTCCTTTGTTCTCCAG-3'. DNA fragments that encompassed intron 1, exon 2, and intron 2 were amplified from $X$. laevis genomic DNA, and then sequenced. The following XRASGRP2 antisense morpholino oligonucleotides (MOs) were designed based on the boundary between exon 1 and intron 1: XRASGRP2a splice inhibition MO (aSMO), 5'-CAGAACTTTAGAAGCCTTACCAAAG-3'; and XRASGRP2b splice inhibition MO (bS-MO), 5'-AGAAATTTAGAACCCATACCGAAGC3'. The MOs were obtained from Gene Tools LLC. The effects of the MOs were confirmed by RT-PCR using the above-mentioned XRASGRP2 common primers. VEGF-A-MO has been described previously (Kalin et al., 2007).

\section{Embryos and microinjection}

Embryonic stage was determined according to the scheme of Nieuwkoop and Faber (Nieuwkoop and Faber, 1994). The jelly coat was removed with Steinberg's solution that contained $4 \%$ cysteine hydrochloride $(\mathrm{pH}$ 8.0). Microinjection was carried out according to the previously described method (Chan et al., 2000). The $\beta$-galactosidase ( $\beta$-gal)mRNA was used as a lineage tracer. The $\beta$-gal-injected embryos were processed for RedGal staining (Research Organics), to reveal $\beta$-galactosidase activity.

\section{Whole-mount in situ hybridization}

Whole-mount in situ hybridization was performed as previously described (Harland, 1991; Abe et al., 2004). Digoxigenin-labeled probes were synthesized from linearized plasmids that encode $X$ flk-1 (Cleaver et al., 1997), Xmsr (Devic et al., 1996), Xtie2 (Iraha et al., 2002), Ami (Inui and Asashima., 2006), XRASGRP2, and globin T3 (Banville and Williams, 1985).

\section{Acknowledgments}

We thank Drs P.A. Krieg, M. Maéno, and I.O. Daar for the generous gifts of plasmids. This work was supported in part by a Grant-in-Aid from the International Cooperative Research Program (ICORP), Japan Science and Technology Agency, and by a Grant-in-Aid for Young Scientists (ST and YO) from the Japan Society for the Promotion of Science (JSPS).

\section{References}

ABE, T., FURUE, M., MYOISHI, Y., OKAMOTO, T., KONDOW, A. and ASASHIMA, M. (2004). Activin-like signaling activates Notch signaling during mesodermal induction. Int J Dev Bio/48: 327-332.

BANVILLE, D. and WILIAMS, J.G. (1985). The pattern of expression of the Xenopus laevis tadpole alpha-globin genes and the amino acid sequence of the three major tadpole alpha-globin polypeptides. Nucleic Acids Res 13: 5407-5421.

BRANNAN, C.I., PERKINS, A.S., VOGEL, K.S., RATNER, N., NORDLUND, M.L., REID, S.W., BUCHBERG, A.M., JENKINS, N.A., PARADA, L.F. and COPELAND N.G. (1994). Targeted disruption of the neurofibromatosis type-1 gene leads to developmental abnormalities in heart and various neural crest-derived tissues. Genes Dev 8:1019-1029.

CHAN, T.C., TAKAHASHI, S. and ASASHIMA, M. (2000). A role for xlim-1 in pronephros development in Xenopus laevis. Dev Bio/228: 256-269.

CHRZANOWSKA-WODNICKA, M., KRAUS, A.E., GALE, D., WHITE, G.C.!a. and VANSLUYS J. (2007). Defective angiogenesis, endothelial migration, proliferation, and MAPK signaling in Rap1b-deficient mice. Blood111: 2647-2656.

CLEAVER, O., TONISSEN, K.F., SAHA, M.S. and KREIG, P.A. (1997).
Neovascularization of the Xenopus embryo. Dev Dyn 210: 66-77

CRITTENDEN, J.R., BERGMEIER, W., ZHANG, Y., PIFFATH, C.L., LIANG, Y., WAGNER, D.D., HOUSMAN, D.E. and GRAYBIEL, A.M. (2004). CaIDAG-GEFI integrates signaling for platelet aggregation and thrombus formation. Nat Med 10: $982-986$

DEVIC, E., PAQUEREAU, L., VERNIER, P., KNIBIEHLER, B. and AUDIGIER, Y. (1996). Expression of a new G protein-coupled receptor X-msr is associated with an endothelial lineage in Xenopus laevis. Mech Dev 59: 129-140.

DOANES, A.M., HEGLAND, D.D., SETHI, R., KOVESDI, I., BRUDER, J.T. and FINKEL T. (1999). VEGF stimulates MAPK through a pathway that is unique for receptor tyrosine kinases. Biochem Biophys Res Commun 255: 545-548.

FERRARA, N., GERBER, H.P. and LECOUTER, J. (2003). The biology of VEGF and its receptors. Nat Med 9: 669-676.

GITLER, A.D., ZHU, Y., ISMAT, F.A., LU, M.M., YAMAUCHI, Y., PARADA, L.F. and EPSTEIN, J.A. (2003). Nf1 has an essential role in endothelial cells. Nat Genet 33: $75-79$.

HARLAND, R.M. (1991). In situhybridization: An improved whole-mount method for Xenopus embryos. Methods Cel/ Bio/36: 685-695.

HENKEMEYER, M., ROSSI, D.J., HOLMYARD, D.P., PURI, M.C., MBAMALU, G., HARPAL, K., SHIH, T.S., JACKS, T. and PAWSON T. (1995). Vascular system defects and neuronal apoptosis in mice lacking ras GTPase-activating protein. Nature 377: 695-701.

HOOD, J.D., FRAUSTO, R, KIOSSES, W.B., SCHWARTZ, M.A. and CHERESH D.A. (2003). Differential alphav integrin-mediated Ras-ERK signaling during two pathways of angiogenesis. J Cell Bio/162: 933-943.

INUI, M. and ASASHIMA, M. (2006). A novel gene, Ami is expressed in vascular tissue in Xenopus laevis. Gene Expr Patterns 6: 613-619.

INUI M., FUKUI A., ITO Y. and ASASHIMA M. (2006). Xapelin and Xmsr are required for cardiovascular development in Xenopus laevis. Dev Bio/298: 188200.

IRAHA, F., SAITO, Y., YOSHIDA, K., KAWAKAMI, M., IZUTSU, Y., DAAR, I.O. and MAENO, M. (2002). Common and distinct signals specify the distribution of blood and vascular cell lineages in Xenopus laevis embryos. Dev Growth Differ 44: 395-407

JOHNSON, L., GREENBAUM, D., CICHOWSKI, K., MERCER, K., MURPHY, E., SCHMITT, E., BRONSON, R.T., UMANOFF, H., EDELMANN, W. KUCHERLAPATI, R. and JACKS, T. (1997). K-ras is an essential gene in the mouse with partial functional overlap with N-ras. Genes Dev 11: 2468-2481.

KALIN, R.E., KRETZ, M.P., MEYER, A.M., KISPERT, A., HEPPNER, F.L. and BRANDLI, A.W. (2007). Paracrine and autocrine mechanisms of apelin signaling govern embryonic and tumor angiogenesis. Dev Bio/305: 599-614.

KAWASAKI, H., SPRINGETT, G.M., TOKI, S., CANALES, J.J., HARLAN, P., BLUMENSTIEL, J.P., CHEN, E.J., BANY, I.A., MOCHIZUKI, N., ASHBACHER, A., MATSUDA, M., HOUSMAN, D.E. and GRAYBIEL, A.M. (1998). A Rap guanine nucleotide exchange factor enriched highly in the basal ganglia. Proc Natl Acad Sci USA 95: 13278-13283.

KOERA, K., NAKAMURA, K., NAKAO, K., MIYOSHI, J., TOYOSHIMA, K., HOTTA T., OTANI, H., AIBA, A. and KATSUKI, M. (1997). K-ras is essential for the development of the mouse embryo. Oncogene 15: 1151-1159.

KOIBUCHI, N., TANIYAMA, Y., NAGAO, K., OGIHARA, T., KANEDA, Y. and MORISHITA, R. (2006). The effect of VEGF on blood vessels and blood cells during Xenopus development. Biochem Biophys Res Commun 344: 339-345.

MEADOWS, K.N., BRYANT, P. and PUMIGLIA, K.M. (2001). Vascular endothelial growth factor induction of the angiogenic phenotype requires Ras activation. $J$ Biol Chem 276: 49289-49298.

MURRAY, P.D.F. (1932). The development in vitro of the blood of early chick embryo. Proc. R.Soc.Lond11: 497-521.

NAGAMINE, K., MATSUDA, A., ASASHIMA, M. and HORI, T. (2008). XRASGRP2 expression during early development of Xenopus embryos. Biochem Biophys Res Commun 372: 886-891.

NIEUKOOP, P.D. and FABER, J. (1994). Normal table of Xenopus laevis (daudin) Garland Publishing Inc, New York.

PASVOLSKY, R., FEIGELSON, S.W., KILIC, S.S., SIMON, A.J., TAL-LAPIDOT, G., GRABOVSKY, V., CRITTENDEN, J.R., AMARIGLIO, N., SAFRAN, M. GRAYBIEL, A.M., RECHAVI, G., BEN-DOR, S., ETZIONI, A. and ALON, R. (2007). A LAD-III syndrome is associated with defective expression of the Rap- 
1 activator CaIDAG-GEFI in lymphocytes, neutrophils, and platelets. JExp Med 204: 1571-1582.

QIAN, X., ESTEBAN, L., VASS, W.C., UPADHYAYA, C., PAPAGEORGE, A.G., YIENGER, K., WARD, J.M., LOWY, D.R., and SANTOS, E. (2000). The Sos1 and Sos2 Ras-specific exchange factors: differences in placental expression and signaling properties. EMBO J19: 642-654.

ROBERTS, D.M., ANDERSON, A.L., HIDAKA, M., SWETENBURG, R.L., PATTERSON, C., STANFORD, W.L. and BAUTCH V.L. (2004). A vascular gene trap screen defines RasGRP3 as an angiogenesis-regulated gene required for the endothelial response to phorbol esters. Mol Cel/ Bio/24: 1051510528.

RODRIGUES C.O., NERLICK S.T., WHITE E.L., CLEVELAND J.L. and KING M.L. (2008). A Myc-Slug (Snail2)/Twist regulatory circuit directs vascular development. Development 135: 1903-1911.
SABIN, F.R. (1920). Studies on the origin of the blood vessels and red blood corpuscles as seen in the living blastoderm of chicks during the second day of the incubation. Contrib Embryo/9: 213-262.

SPRINGETT, G.M., KAWASAKI, H. and SPRIGGS, D.R. (2004). Non-kinase second-messenger signaling: new pathways with new promise. Bioessays 26 : 730-738.

TURNER, D.L. and WEINTRAUB, H. (1994). Expression of achaete-scute homolog 3 in Xenopus embryos converts ectodermal cells to a neural fate. Genes Dev8: 1434-1447.

WANG, D.Z., HAMMOND, V.E., ABUD, H.E., BERTONCELLO, I., MCAVOY, J.W and BOWTELL D.D. (1997). Mutation in Sos1 dominantly enhances a weak allele of the EGFR, demonstrating a requirement for Sos1 in EGFR signaling and development. Genes Dev 11: 309-320.

\section{Further Related Reading, published previously in the Int. J. Dev. Biol.}

See our recent Special Issue Epigenetics \& Development edited by Saadi Khochbin and Stefan Nonchev at:

http://www.ijdb.ehu.es/web/contents.php?vol=53\&issue=2-3

Lef1 plays a role in patterning the mesoderm and ectoderm in Xenopus tropicalis

Giulietta Roël, Yoony Y.J. Gent, Josi Peterson-Maduro, Fons J. Verbeek and Olivier Destrée

Int. J. Dev. Biol. (2009) 53: 81-89

Building the vertebrate heart - an evolutionary approach to cardiac development José M. Pérez-Pomares, Juan M. González-Rosa and Ramón Muñoz-Chápuli

Int. J. Dev. Biol. (2009) 53: 1427-1443 (doi: 10.1387/ijdb.072409jp)

Embryonic development of the proepicardium and coronary vessels

Anna Ratajska, Elzbieta Czarnowska and Bogdan Ciszek

Int. J. Dev. Biol. (2008) 52: 229-236

An activating mutation in the PDGF receptor-beta causes abnormal morphology in the mouse placenta Camilla Looman, Tong Sun, Yang Yu, Agata Zieba, Aive Ahgren, Ricardo Feinstein, Henrik Forsberg, Carina Hellberg, Carl-Henrik Heldin, Xiao-Qun Zhang, Karin Forsberg-Nilsson, Nelson Khoo, Reinald Fundele and Rainer Heuchel

Int. J. Dev. Biol. (2007) 51: 361-370

Blood vessel/epicardial substance (bves) expression, essential for embryonic development, is down regulated by Grk/EFGR signalling

Shengyin Lin, Debiao Zhao and Mary Bownes

Int. J. Dev. Biol. (2007) 51: 37-44

Generation of the germ layers along the animal-vegetal axis in Xenopus laevis $\mathrm{H}$ Yasuo and $\mathrm{P}$ Lemaire

Int. J. Dev. Biol. (2001)‘45: 229-235

Ets-1 and Ets-2 proto-oncogenes exhibit differential and restricted expression patterns during Xenopus laevis oogenesis and embryogenesis

D Meyer, M Durliat, F Senan, M Wolff, M Andre, J Hourdry and P Remy

Int. J. Dev. Biol. (1997) 41: 607-620

Whole-mount in situ hybridization reveals the expression of the XI-Fli gene in several lineages of migrating cells in Xenopus embryos

D Meyer, P Stiegler, C Hindelang, A M Mager and P Remy

Int. J. Dev. Biol. (1995) 39: 909-919

5 yr ISI Impact Factor $(2008)=3.271$

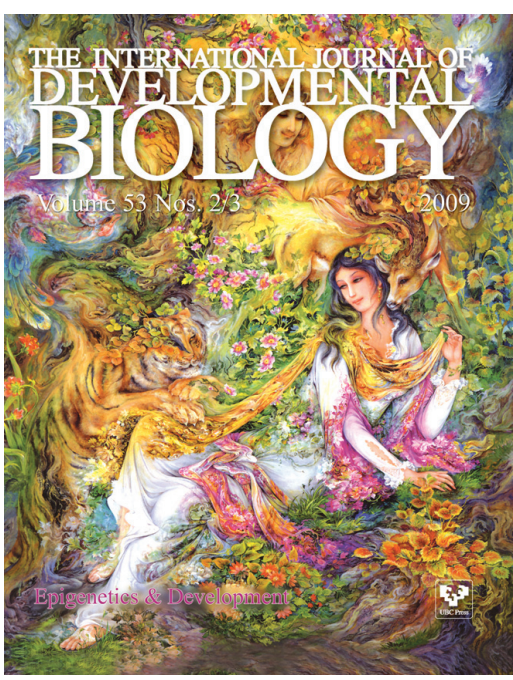

